\title{
Clinical value of postoperative sentinel lymph node biopsy
}

\author{
Zhixian $\mathrm{He}^{1 \#}$, Yi Zhou ${ }^{2 \#}$, Feiran Wang ${ }^{1}$, Qian $\mathrm{Xu}^{1}$, Wei Zhang ${ }^{1}$, Xiaojian $\mathrm{Ni}^{3}$, Sujie $\mathrm{Ni}^{4}$ \\ ${ }^{1}$ Department of General Surgery, ${ }^{2}$ Department of General Surgery, The First Affiliated Hospital of Nanjing Medical University, Nanjing 210000 , \\ China; ${ }^{3}$ Department of General Surgery, Fudan University Zhongshan Hospital, Shanghai 200000, China; ${ }^{4}$ Department of Medical Oncology, \\ Affiliated Hospital of Nantong University, Nantong 226000, China \\ Contributions: (I) Conception and design: S Ni, Z He; (II) Administrative support: S Ni; (III) Provision of study materials or patients: Z He, \\ W Zhang, F Wang, Q Xu; (IV) Collection and assembly of data: F Wang, Q Xu, Y Zhou; (V) Data analysis and interpretation: Y Zhou, S Ni, Z He; (VI) \\ Manuscript writing: All authors; (VII) Final approval of manuscript: All authors. \\ \#These authors contributed equally to this work. \\ Correspondence to: Sujie Ni. Department of Medical Oncology, Affiliated Hospital of Nantong University, Nantong 226000, China. \\ Email: nisujie@fudan.edu.cn; Xiaojian Ni. Department of General Surgery, Fudan University Zhongshan Hospital, Shanghai 200000, China. \\ Email: nixiaojian02010630@126.com.
}

Background: This study analyzed the clinical data and general information of breast cancer patients who were admitted by the Affiliated Hospital of Nantong University and underwent lumpectomy, followed by sentinel lymph node biopsy (SLNB) to investigate the effect of tumor location on the sentinel lymph node (SLN) detection rate, obtain a clear understanding of the SLNB procedure and further promote the use of this procedure in the local area.

Methods: This study involved a total of 118 patients who were diagnosed with breast cancer and admitted by the Affiliated Hospital of Nantong University for lumpectomy and SLNB between July 2015 and June 2019. An analysis was conducted to explore the role of tumor location in the detection of SLNs.

Results: Tumor location was associated with the success rate of post-lumpectomy SLNB. In the case of tumor location in the upper outer quadrant (UOQ) of the breast near the axilla, the SLN detection rate was relatively low. In contrast, when a tumor occurred in any of the other quadrants or the UOQ next to the areola, the tumor location had no significant impact on the SLN detection rate. SLNB indicated that 102 out of the 118 patients had SLNs, with the detection rate of $86.4 \%$. Particularly, for patients whose tumors were located in the UOQ near their axillae, the SLN detection rate was 30\% (3/10). As to tumor location in other quadrants or the UOQ next to the areola, the SLN detection rate was up to $90.8 \%(99 / 109)$.

Conclusions: The performance of post-lumpectomy SLNB is associated with tumor location. SLNB is recommended when the tumor site lies in the upper inner/lower outer/lower inner quadrants (UIQ/LOQ/ LIQ) of the breast or the UOQ next to the areola. If the SLNB result turns out to be negative, there is no need to perform axillary lymph node dissection (ALND). For tumor location in the UOQ of the breast, especially when it is near the axilla, SLNB is not a favorable option after lumpectomy. It is recommended that the patient receive a core needle biopsy (CNB) before SLNB.

Keywords: Breast cancer; post-lumpectomy; sentinel lymph node biopsy (SLNB)

Submitted Oct 13, 2019. Accepted for publication Nov 05, 2019.

doi: 10.21037/atm.2019.11.106

View this article at: http://dx.doi.org/10.21037/atm.2019.11.106 


\section{Introduction}

Breast cancer is now one of the greatest threats to women's health. Reportedly, breast cancer is the most frequently diagnosed malignancy among women, and the incidence rate keeps increasing in China (1). Despite the continuous development of genetic research, studies on targeted therapy, immune regulation and anticancer therapy, and breast cancer treatment, surgical procedures remain the mainstay of treatment for breast cancer. Since the emergence of classic mastectomy and ALND, these procedures have been an important treatment for breast cancer for a long time (2). However, a series of complications arise after mastectomy and ALND, which severely affects patients' quality of life, such as surgical scars, pain, and a shoulder movement disorder. With medical technology developing and medical models being transformed, psychological defects and other psychosocial problems facing breast cancer patients have become a major concern.

Over time, we have gained a better understanding of breast cancer. In the 1970s, an American researcher Dr. Fisher proposed that breast cancer was a systemic disease, and the overall survival would not be affected by the management of primary lesions and local lymph nodes, regardless of the approaches and regimens. With lymph nodes being the most common site of metastases in breast carcinoma, axillary lymph node status is an independent prognostic variable in the management of patients with breast cancer, making lymph node dissection an essential part of breast cancer treatment. Previous studies reported the first research on SLNs in patients with breast cancer using $99 \mathrm{mTc}-\mathrm{SC}$ as a tracer in the preliminary study (3). Some results of studies provided by Halsted and Fisher (4) proposed that breast cancer was not systemic at the onset but became a systemic disease when cancer spread to lymph nodes, which has laid a foundation for further study on SLNs. As the SLN tracing and positioning techniques and the biopsy technology develop, the SLNB procedure is established according to the characteristics of SLN drainage and the progression of breast cancer. Besides, some previous articles relevant articles for systematic evaluation and analysis came to a conclusion that SLNB was a superior technique for axillary lymph node staging in breast cancer as it allowed for accurate assessment of axillary lymph node status and prevented a series of sequelae resulting from unnecessary ALND (5). Later, multiple clinical trials, including the ALMANAC trial (UK) and the
NSABP B-32 trial (US), have proved the accuracy of SLNB in axillary lymph node staging; SLNB and ALND have the same axillary lymph node-positive rate and produce similar outcomes in terms of 5 -year survival and tumorfree survival $(6,7)$. As researchers from all around the world engage in the study of SLNB's success rate, accuracy, sensitivity, negative predictive value, and false-negative rate, there is ample evidence showing that SLNB is highly accurate and has a tolerable false-negative rate. In recent years, identification of SLN location has been applied to the diagnosis and treatment of tumors in other body parts, such as tumors in the head and the neck, gastrointestinal cancer, gynecologic cancer, genitourinary cancer, and lung cancer. In western countries, SLNB is now a regular procedure used for diagnosis and treatment of malignant melanoma, as well as an important criterion for the pathological staging of the disease (8).

As a late starter in the field, China makes slow progress in SLN research for many reasons. First, China has a lower level of economic and medical development compared to the developed countries; second, there are strict restrictions on the use of dyes and radionuclides; third, relevant equipment is extremely expensive and gives serious cause for concern of nuclear radiation; fourth, considering the necessary interdisciplinary collaboration between nuclear medicine and pathology, during the early stage, SLN research is only feasible and practical in large $3 \mathrm{~A}$ hospitals. Moreover, since there is no established standard for the use of relevant techniques but enormous concern about the negative impact of the false-negative rate and a lack of medical and regulatory support for domestic SLN research, relevant research data are scarcely available. Reports on domestic SLN studies emerge since 2000. The CBCSG-001 trial (China multicenter study of sentinel node biopsy substituting axillary node dissection) commenced in January 2002, and the recruitment of participants ended in June 2007. In the study, SLNB yielded an overall 5 -year survival rate of $98.2 \%$ and a disease-free survival rate of $94.2 \%$, basically in agreement with the research data provided by international trials (9). In clinical practice, considering the differences in regions and medical ideas, secondary hospitals or some tertiary hospitals in the underdeveloped areas tend to remove tumors and perform SLNB after pathological examination rather than order CNB before lumpectomy. Some patients even receive Mammotome resection. This study also elaborated on the cases where pathological examinations indicated malignancy to discuss whether the patients were eligible for SLNB and whether lymph vessels 
were damaged in relevant surgical procedures because of tumor location.

\section{Methods}

This study involved 118 female patients who were diagnosed with breast cancer and admitted by the Affiliated Hospital of Nantong University during July 2015 and June 2019. Considering the definite pathological diagnosis of malignancy, these patients had received lumpectomy before SLNB. The mean age was 54.7 (range: 29-73). There were 57 cases of left-breast cancer and 61 cases of right-breast cancer, including 48 in the UOQ, 19 in the LOQ, 24 in the UIQ, 17 in the LIQ, and 10 in the central portion of their breasts. More specifically, 10 tumors in the UOQ were next to the axilla, another 38 in the UOQ were next to the areola.

According to the TNM staging of breast cancer, 92 patients had stage $\mathrm{T} 1$ breast cancer, and 26 had stage $\mathrm{T} 2$ breast cancer. Nine patients underwent Mammotome biopsy before operation while 109 patients received regular lumpectomy. Postoperative histology: 80 cases of infiltrating ductal carcinoma (IDC), 23 ductal carcinomas in situ (DCIS), 3 mucinous carcinomas (MC), and 12 others.

\section{Inclusion criteria}

(I) Female;

(II) no enlargement of axillary nodes in preoperative B-scan ultrasonography or molybdenum target radiography;

(III) no neoadjuvant chemotherapy or radiotherapy before the operation, no history of surgery or radiotherapy on the axilla;

(IV) no contraindications of SLNB.

\section{Exclusion criteria}

(I) Male;

(II) indications of enlargement of axillary nodes or metastasis in preoperative examinations;

(III) multicentric breast cancer;

(IV) allergy to tracer;

(V) other contraindications of SLNB.

\section{Primary Instruments and Reagents}

One percent coeruleum methylene (methylene blue, $\mathrm{MB}$ ) manufactured by Jiangsu Jichuan Pharmaceutical Co., Ltd.

\section{Research methods}

\section{Surgical procedures}

Each patient was asked to lie in the supine position, with the affected upper limb extended. About $2 \mathrm{~mL} \mathrm{1 \%}$ coeruleum methylene was injected subcutaneously into the subareolar area surrounding the lesion, followed by a local massage for 5-10 min until coeruleum methylene was completely absorbed by lymph vessels. An arc incision (3-4 cm) was made near the axilla $10-15$ min later. The incision was exposed to identify the stained lymph vessels or lymph nodes. SLNs were located by tracing the stained lymph nodes along the axilla, which was then resected for pathological biopsy. If no stained SLNs were found, another incision should be made. If no stained SLNs were identified in the new incision, it should be considered that the SLNB result was negative and ALND should be performed.

Intraoperative decisions should be made based on realities. In case of breast-conserving lumpectomy, the tumor bed and the peripheral breast tissue about $1 \mathrm{~cm}$ around the tumor bed should be dissected, with the upper, lower, inner, outer, and basal incisal edges being labeled explicitly to ensure negativity of surgical margins; if two surgical margins were positive, the surgeon should drop the breast-conserving procedure and remove the affected breast. After preparing a specimen of the dissected breast, locating marks (4-6 titanium clips) for tumor bed boost radiotherapy should be placed in the operative field after rinsing (note: the patient should be informed of postoperative radiotherapy through effective communication before operation). In the case of mastectomy, a transverse fusiform incision should be made about $5 \mathrm{~cm}$ away from the tumor edge, with the length of the incision depending on the size of the affected breast and the location of the nipple. When removing the entire breast and the surrounding fat tissue, the skin should be separated from the superficial fascia, with a moderate amount of subcutaneous fat of the skin flap leaving intact.

\section{Pathological examination}

Any blue-stained lymph nodes that were found during the operation were definitely SLNs and should be identified as lymph nodes through pathological verification.

\section{Postoperative adjuvant therapy}

Breast-conserving lumpectomy or mastectomy was 
performed based on each patient's condition. Meanwhile, chemotherapy, radiotherapy, endocrine therapy, and targeted therapy were given according to pathological findings, IHC test results, and relevant diagnosis and treatment protocols after thorough communication with the patients.

\section{Statistical analysis}

All enumeration data were examined using the chi-squared test or Fisher's exact test. Generally, $\mathrm{P}<0.05$ indicated a difference in statistical significance. The software SPSS 20.0 was used for data analysis.

\section{Results}

\section{SLN yield and detection rate}

Among the 118 patients, SLNs were detected in 102 patients and were not successfully detected in another 16 patients. The SLN detection rate was $86.4 \%(102 / 118)$. For the 10 patients having primary breast cancer in the UOQ near the axilla, SLN detection was successful in 3 cases, representing a detection rate of $30 \%(3 / 10)$. For the 38 patients with primary breast cancer in the UOQ near the areola, SLNs were successfully detected in 33 patients, indicating a detection rate of $86.8 \%(33 / 38)$. Specifically, SLN detection was successful in all the 17 cases of breast cancer in the LIQ (detection rate $=100 \%$ ); SLNs were detected in 23 out of the 24 patients with breast cancer in the UIQ (detection rate $=95.8 \%$ ); 17 out of the 19 patients with breast cancer in the LOQ failed the SLN detection (detection rate $=89.5 \%$ ) and 9 out of the 10 patients with breast cancer in areola failed the SLN detection (detection rate $=90 \%$ ).

\section{SLN detection rate and relevant factors}

SLN detection was successfully in 102 out of the 118 patients, with a success rate of $86.4 \%$. Among all factors, whether a tumor occurs in the left or the right breast, age, primary tumor size, BMI, and pathological type is not associated with the success rate of SLN detection (chisquared test, $\mathrm{P}>0.05$ ) whereas tumor location is correlated with the SLN detection rate $(\mathrm{P}<0.05)$. Through in-depth analysis, the SLN detection rate was $86.8 \%$ when breast tumors were located in the UOQ near the axilla, much lower than the SLN detection rates when breast cancer occurred in other quadrants. For patients with breast cancer in the UOQ near the areola, the SLN detection rate was $30 \%$. The SLN detection rates in the LIQ, UIQ, and LOQ groups were $100 \%, 95.8 \%$, and $89.5 \%$, respectively. SLN detection rate was $90 \%$ in the case of tumor location in the central portion of the breast. See Table 1, Figure 1.

\section{Discussion}

With the vigorous development of medical technology, diverse approaches are introduced to treat breast cancer. Despite all that, surgical intervention is still the mainstay of treatment for patients with breast cancer (10). SLNB brings the surgical treatment of breast cancer to a new era. Through clinical promotion and application, SLNB helps breast cancer patients without metastatic axillary nodes avoid unnecessary ALND and a series of postoperative complications, thereby improving their quality of life (11). The introduction of SLNB in breast cancer treatment is a clear manifestation of the modern medical model of bringing greater benefits to patients with technology.

The lymphatic system of the breasts is rather complicated. Lymph flows back to the subareolar lymphatic vessels or the lymphatic plexus around the nipple through the superficial lymph vessels. These flapless lymph vessels and the dermal lymphatics are interconnected and merged into the Sappey subareolar plexus. Also, they are connected to other subcutaneous and dermal lymphatics through vertical lymph vessels. Lymph flows in one direction from the superficial to the subcutaneous layers and from the subareolar plexus to the perilobular areas and the subdermal plexus. Periductal lymph vessels are located in the epitheliomuscular layer on the vessel walls. Lymph flows from the deep subcutaneous layer and the internal mammary lymphatics to the axilla and the internal mammary lymph nodes (IMLNs) $(12,13)$.

In terms of breast anatomy, sentinel lymph nodes (SLNs) are frontier lymph nodes for lymph collection in an organ or tissue. Histopathology of SLNs from an area can reflect the lymph node status in the area (14). A positive SLNB result suggests that cancer has already spread to the lymph nodes in that area. Research on SLNs offers new insights into the physiological mechanism of breast lymph node anatomy and reflux. A study argues that the lymph in skin and glands flows back to the same lymph nodes, which are also primary destinations of mammary lymph. Another study on lymphoscintigraphy reveals that the lymph in deep glands or retromammary lymph nodes first returns to the IMLNs 
Table 1 Influencing factors of SLNB

\begin{tabular}{|c|c|c|c|c|}
\hline Parameter & $\mathrm{n}$ & Sentinel lymph node & $\chi^{2}$ & $\mathrm{P}$ value \\
\hline Age & & & 0.001 & 0.978 \\
\hline$>50$ & 66 & 57 & & \\
\hline$\leq 50$ & 52 & 45 & & \\
\hline $\mathrm{BMI}>30$ & 5 & 3 & & \\
\hline $\mathrm{BMI}<30$ & 113 & 99 & & \\
\hline \multicolumn{5}{|l|}{ Side of primary tumor } \\
\hline Left side & 57 & 53 & 4.026 & $0.045^{*}$ \\
\hline $\mathrm{T} 1$ & 92 & 81 & & \\
\hline $\mathrm{T} 2$ & 26 & 21 & & \\
\hline Location of tumor & & & 31.914 & $<0.001^{*}$ \\
\hline UOQ (next to the axilla) & 10 & $3(30 \%)$ & & \\
\hline UOQ (next to the areola) & 38 & $33(86.8 \%)$ & & \\
\hline LOQ & 19 & $17(89.5 \%)$ & & \\
\hline UIQ & 24 & $23(95.8 \%)$ & & \\
\hline LIQ & 17 & $17(100 \%)$ & & \\
\hline Others & 12 & 11 & & \\
\hline
\end{tabular}

*, $\mathrm{P}$ value $<0.05$ was statistically significant differences. SLNB, sentinel lymph node biopsy.

through subcutaneous or intracutaneous lymph vessels (15). According to the Berg muscle-based categorization of axillary lymph node location, axillary lymph drainage of the mammary glands proceeds in the following order: level I $\rightarrow$ level II $\rightarrow$ level III. The spread of breast cancer cells basically follows the same route. In a few cases, metastatic lymph nodes are found in level II or III when the lymph nodes in level I are not involved. Anatomic studies show that $75 \%$ of the lymph flows back to the axillary nodes and the rest $25 \%$ to the IMLNs, which causes crosslevel metastasis through the IMLNs. Axillary lymph node metastasis in breast cancer generally follows a top-down pattern. In western countries, there is a long history of SLN research. Some reports showed the first research on SLNs in patients with breast cancer using $99 \mathrm{mTc}-\mathrm{SC}$ as a tracer in the preliminary study (3). Another reports indicated (4) combined the study results provided by Halsted and Fisher and put forward that breast cancer was limited in the affected breast at onset and spread to the entire body through metastatic lymph nodes, which has laid a foundation for further study on SLNs. As the SLN tracing and positioning techniques and the biopsy technology 


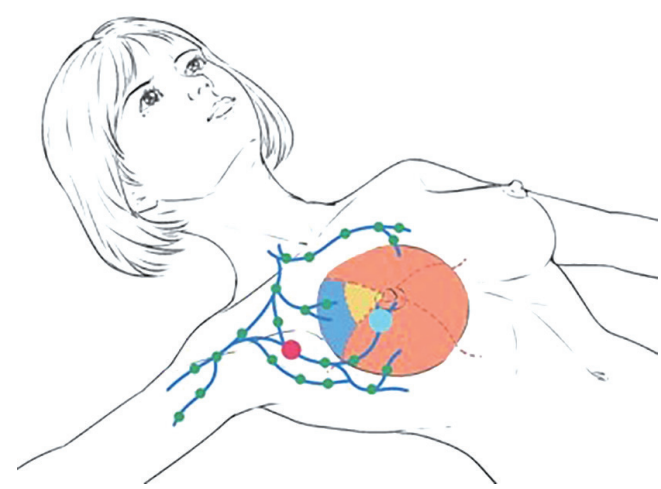

Figure 1 Drawings illustrate different tumor locations and lymphatic vessels in the breast.

develop, the SLNB procedure is established according to the characteristics of SLN drainage. In 2000, a foreign study gathered 18 relevant articles for systematic evaluation and analysis and came to a conclusion that SLNB was a superior technique for axillary lymph node staging in breast cancer as it allowed for accurate assessment of axillary lymph node status and prevented a series of sequelae resulting from unnecessary ALND (5). Later in the ALMANAC trial (UK) and the NSABP B-32 trial (US), it was demonstrated that SLNB can be used for accurate axillary lymph node staging, and relevant patients had no significant difference in the 5 -year survival and tumor-free survival $(8,9)$.

Presently, SLNB is a standard surgical intervention for breast cancer. Generally, SLNB is performed before operation on the breast. In clinical practice, since there are huge differences in regions, economic development level, and publicity of medical knowledge, secondary hospitals in some areas seldom use CNB before operation; besides, because CNB imposes a risk of spreading tumor cells through needle-track metastasis, many patients tend to receive lumpectomy and decide the following surgical plan and whether they are eligible for SLNB according to the indications given in the pathological examination. Hence, this trial was conducted to analyze and evaluate the results.

Among the 118 patients, SLNs were detected in 70 patients and were not successfully detected in another 11 patients. The SLN detection rate was $86.4 \%$ (102/118). For the 10 patients having primary breast cancer in the UOQ near the axilla, SLN detection was successful in 3 cases, representing a detection rate of $30 \%(3 / 10)$. For the 38 patients with primary breast cancer in the UOQ near the areola, SLNs were successfully detected in 33 patients, indicating a detection rate of $86.8(33 / 38)$. Specifically,
SLN detection was successful in all the 17 cases of breast cancer in the LIQ (detection rate $=100 \%$ ); SLNs were detected in 23 out of the 24 patients with breast cancer in the UIQ (detection rate $=95.8 \%$ ); 17 out of the 19 patients with breast cancer in the LOQ failed the SLN detection (detection rate $=89.5 \%$ ). The UOQ group had an SLN detection rate significantly lower than the other three groups. To eliminate other variables, the patients were divided into two groups by tumor location, with one group having tumors in the UOQ near the axilla and the other with tumors in the non-UOQ near the axilla. Through comparison using the chi-squared test, the difference between the two groups had statistical significance $\left(\chi^{2}=31.914, \mathrm{P}<0.001\right)$.

There are numerous factors that influence the success rate of SLNB, such as learning curve, age, BMI, tumor size, tumor location, and pathological grading (16). In this study, relevant analyses were different from previous studies. Based on the characteristics of SLN drainage and clinical practices, the present study found that SLN detection failed in 3 out of the 10 patients with tumors in the UOQ next to the Axilla of their breasts, indicating a failure rate higher than the other groups. This is probably because intratumoral lymph vessels were damaged in the initial operation. Also, it is believed that lumpectomy is associated with the level of damage to the lymphatic system. Results of the cause factor analysis are as follows:

(I) Selection of tracer and injection site. Presently, SLNB is performed with the vital stain method, the radionuclide method, or the combined method. Generally, the radionuclide method shows a higher level of accuracy compared to the vital stain method. In spite of the high accuracy, this method requires professional instruments and reagents, and the SLNB procedure is relatively complicated. The deep concern about radioactivity also limits its clinical application. The vital stain method is cost-effective and easy-to-use. As no additional instruments or reagents are required, this method is widely used in China. The success rate of SLNB using the vital stain method depends on the selection of dyes and surgical techniques. In foreign countries, the commonly used vital stains include isosulfan blue (IB), patent blue (PB), and methylene blue (MB). Despite the advantages of high selectivity and absorptivity, IB and $\mathrm{PB}$ are relatively expensive and require approval from domestic authorities before use. All this makes 
MB the most commonly used tracer in China. It has been demonstrated in multiple trials using coeruleum methylene for lymph node tracing in breast cancer can produce satisfactory outcomes in terms of detection rate, sensitivity, and falsenegative rate (17). Some researchers used the combined method for SLNB in multicentric breast cancer and reached a conclusion that injection sites had an insignificant effect on the accuracy of SLNB and the anatomic location of SLNs (18). In this clinical trial, $1 \%$ coeruleum methylene (methylene blue, MB) manufactured by Jiangsu Jichuan Pharmaceutical Co., Ltd. was injected into all patients. All blue-stained lymph nodes were clear and easy-to-identify with the boundaries between these lymph nodes and the peripheral tissue. Besides, no allergic reaction or toxic side effect was observed. Satisfactory results were yielded by injecting the vital stain into the peritumoral and the subareolar areas.

(II) Obesity: a foreign study showed that obese patients faced a high risk of edema in their upper limbs after ALND and thus considered obesity an indication of SLNB (19). Yet, it is reported that an obese individual (BMI >30) has fewer SLNs, which is probably because adipose tissue takes place of axillary nodes and envelopes the axillary nodes, thereby reducing lymphatic drainage. In addition, it is difficult to perform on obese patients and identify lymph nodes in given areas (20). In this study, there were 5 patients with a BMI exceeding 30 and 113 patients with a BMI below 30, and the comparison between these two groups showed no significant difference. If a larger sample size were available, the findings might be consistent with the previous studies.

(III) Age: it is reported that in patients at the age of 50 and above, the SLN detection rate is relatively low. In breast cancer patients at an advanced age, the lymphatic tissue in lymph nodes has degenerated while the lymph nodes function less efficiently to engulf pathogens, act as an innate barrier, and absorb and transport fatty acids and fats. In the meantime, the hydrostatic pressure becomes lower. As a result, the lymph nodes are less efficient in stain absorption, aggregation, and transportation, leading to a lower detection rate of SLNs (21). In this trial, no clear correlations were identified between age and the SLN detection rate probably because each age group was composed of a limited number of patients.

(IV) Tumor size: there is evidence showing that lymph node metastasis is associated with tumor size, which is considered as a predictive variable for metastasis (22). In this study, more tissue and lymph vessels were destroyed when collecting specimens from larger tumors. In this case, the SLN detection rate became lower because of reduced dye aggregation in the SLNs. The patients were divided into two groups by tumor size, and the difference between the two groups was not significant. This is probably because of the small sample size and the lack of stage T3 cases in this trial. The conclusion may be consistent with previous findings when the larger sample size is used or patients in stage $\mathrm{T} 3$ are recruited in the study.

(V) Tumor location: according to the characteristics of breast lymphatic drainage, if a tumor is located in the UOQ of the breast near the axilla and SLNs where there are numerous draining lymph vessels, the SLN detection rate may be affected as the lumpectomy may bring damage to the lymph vessels. If a tumor is located in the inner quadrant near the IMLNs, there is a high risk of metastasis to the IMLNs. In this trial, the SLN detection rate was relatively low in patients with tumors in the UOQ. Through careful analysis of the original data, SLNs were successfully detected in 36 patients, and compared to the rest 33 cases, the SLNs were closer to the nipple. In other words, the SLN detection failed in the 12 cases probably because the tumors were extremely close to the axilla where the lymphatic system was damaged. In the UIQ, LOQ, and LIQ groups, the detection rates were almost the same as those of normal mammary glands.

(VI) Internal mammary lymph node (IMLN): It is generally believed that tumors in the UIQ of the breast are closer to the internal mammary lymph chain, which indicates a higher risk of metastasis to the IMLNs. In terms of metastasis of breast cancer, metastasis to the IMLNs is secondary to that of the axillary nodes. The metastasis of breast cancer is an important basis for clinical staging, adjuvant therapy, and prognosis (23). Taking IMLN metastasis into consideration in breast 
cancer staging provides more reliable evidence for adjuvant therapy. With the publicity of SLNB, IMLN dissection becomes a promising procedure for breast cancer treatment as it causes less trauma than ALND. In this trial, only 1 case of breast cancer in the UIQ failed SLN detection. The SLN detection rate of the LIQ group was $100 \%$. There was no significant difference between the two groups. Therefore, no clear evidence shows any correlations between IMLN metastasis and SLN metastasis. This is because the present study has a limited sample size. Hence, larger sample size is needed for further study.

(VII) Learning curve: in domestic and foreign studies, the success rate of SLNB in breast cancer is closely associated with the time of learning relevant techniques. The SLNB learning curve shows that the SLN detection rate is related to biopsy operators' clinical techniques (11).

\section{Conclusions}

In conclusion, we found that the performance of postlumpectomy SLNB is associated with tumor location. Postlumpectomy SLNB is feasible when the tumor site lies in the UIQ/LOQ/LIQ of the breast or the UOQ next to the areola. If the SLNB result turns out to be negative, there is no need to perform ALND. If you want to perform SLNB for the patients whose tumor location in the UOQ of the breast, especially when it is near the axilla, lumpectomy is not a favorable option. These results should improve our understanding of the SLNB procedure and further promote the use of this procedure in the local area.

\section{Acknowledgments}

Funding: This work was supported by grants from the National Natural Scientific Funding (81702608) to NSJ, Science and Technology Project of Nantong (MS12017007-1) to HZX and Shanghai top priority Clinical Medical Center and Key Discipline Construction Plan (2017ZZ02007).

\section{Footnote}

Conflicts of Interest: The authors have no conflicts of interest to declare.

Ethical Statement: The authors are accountable for all aspects of the work in ensuring that questions related to the accuracy or integrity of any part of the work are appropriately investigated and resolved. This study was approved by Ethics the Committee of Affiliated Hospital of Nantong University (ID: 2013-67). Informed consent was provided by all participants.

\section{References}

1. Torre LA, Trabert B, Desantis CE, et al. Ovarian cancer statistics, 2018. CA Cancer J Clin 2018;68:284-96.

2. Giuliano AE, Ballman K, McCall L, et al. Locoregional Recurrence After Sentinel Lymph Node Dissection With or Without Axillary Dissection in Patients With Sentinel Lymph Node Metastases: Long-term Follow-up From the American College of Surgeons Oncology Group (Alliance) ACOSOG Z0011 Randomized Trial. Ann Surg 2016;264:413-20.

3. Gandhi SJ, Satish C, Shanmuga Sundaram P, et al. Axillary reverse mapping using 99mTc-SC: a case illustration. Clin Nucl Med 2014;39:e428-30.

4. Halsted CP, Benson JR, Jatoi I. A historical account of breast cancer surgery: beware of local recurrence but be not radical. Future Oncol 2014;10:1649-57.

5. Motomura K. Sentinel node biopsy for breast cancer: past, present, and future. Breast Cancer 2015;22:212-20.

6. Mansel RE, Fallowfield L, Kissin M, et al. Randomized multicenter trial of sentinel node biopsy versus standard axillary treatment in operable breast cancer: the ALMANAC Trial. J Natl Cancer Inst 2006;98:599-609.

7. Julian TB, Anderson SJ, Krag DN, et al. Abstract S2-05: 10-yr follow-up results of occult detected sentinel node disease: NSABP B-32, a randomized phase III clinical trial to compare sentinel node resection (SNR) to conventional axillary dissection $(\mathrm{AD})$ in clinically node-negative breast cancer. Cancer Res 2013;73:Abstract nr S2-05.

8. Wong SL, Faries MB, Kennedy EB, et al. Sentinel Lymph Node Biopsy and Management of Regional Lymph Nodes in Melanoma: American Society of Clinical Oncology and Society of Surgical Oncology Clinical Practice Guideline Update. J Clin Oncol 2018;36:399-413.

9. Lee SA, Lee HM, Lee HW, et al. Risk Factors for a FalseNegative result of Sentinel Node Biopsy in Patients with Clinically Node negative Breast Cancer. Cancer Res Treat 2018;50:625-33.

10. Matsen CB, Neumayer LA. Breast cancer: a review for the general surgeon. JAMA Surg 2013;148:971-9.

11. Qiu SQ, Zhang GJ, Jansen L, et al. Evolution in sentinel 
lymph node biopsy in breast cancer. Crit Rev Oncol

Hematol 2018;123:83-94.

12. Yuan Q, Wu G, Xiao SY, et al. Identification and Preservation of Arm Lymphatic System in Axillary Dissection for Breast Cancer to Reduce Arm Lymphedema Events: A Randomized Clinical Trial. Ann Surg Oncol 2019;26:3446-54.

13. Lopez ME, Olutoye OO. Breast Embryology, Anatomy, and Physiology. In: Ledbetter D, Johnson P. Editors. Endocrine Surgery in Children. Heidelberg: Springer, 2018:365-76.

14. Land SR, Kopec JA, Julian TB, et al. Patient-reported outcomes in sentinel node-negative adjuvant breast cancer patients receiving sentinel-node biopsy or axillary dissection: National Surgical Adjuvant Breast and Bowel Project phase III protocol B-32. J Clin Oncol 2010;28:3929-36.

15. Shahar KH, Buchholz TA, Delpassand E, et al. Lower and central tumor location correlates with lymphoscintigraphy drainage to the internal mammary lymph nodes in breast carcinoma. Cancer 2005;103:1323-9.

16. Lyman GH, Temin S, Edge SB, et al. Sentinel lymph node biopsy for patients with early-stage breast cancer: American Society of Clinical Oncology clinical practice

Cite this article as: $\mathrm{He}$ Z, Zhou Y, Wang F, Xu Q, Zhang W, Ni X, Ni S. Clinical value of postoperative sentinel lymph node biopsy. Ann Transl Med 2019;7(22):683. doi: 10.21037/ atm.2019.11.106 guideline update. J Clin Oncol 2014;32:1365-83.

17. Li H, Jun Z, Zhi-Cheng G, et al. Factors that affect the false negative rate of sentinel lymph node mapping with methylene blue dye alone in breast cancer. J Int Med Res 2019;47:4841-53.

18. Asadi M, Krag D. Internal mammary sentinel lymph node biopsy in clinical practice. Int J Surg 2016;36:332-4.

19. Helyer LK, Varnic M, Le LW, et al. Obesity is a risk factor for developing postoperative lymphedema in breast cancer patients. Breast J 2010;16:48-54.

20. Gawlick U, Mone MC, Nelson ET, et al. Success in sentinel lymph node procedures in obese patients with breast cancer. Am J Surg 2010;200:707-10; discussion 710-1.

21. Murawa D, Hirche C, Dresel S, et al. Sentinel lymph node biopsy in breast cancer guided by indocyanine green fluorescence. Br J Surg 2009;96:1289-94.

22. Liu C, Zhao Z, Gu X, et al. Establishment and Verification of a Bagged-Trees-Based Model for Prediction of Sentinel Lymph Node Metastasis for Early Breast Cancer Patients. Front Oncol 2019;9:282.

23. Urano M, Denewar FA, Murai T, et al. Internal mammary lymph node metastases in breast cancer: what should radiologists know? Jpn J Radiol 2018;36:629-40. 